EPJ Web of Conferences 52, 01008 (2013)

DOI: 10.1051/epjconf/20135201008

(C) Owned by the authors, published by EDP Sciences, 2013

\title{
Leading Hadron Production at HERA
}

\author{
Armen Buniatyan ${ }^{1, a}$ \\ ${ }^{1}$ Deutsches Elektronen Synchrotron, DESY \\ Notkestrasse 85, 22607 Hamburg, Germany
}

\begin{abstract}
Data from the recent measurements of very forward baryon and photon production with the H1 and ZEUS detectors at electron-proton collider HERA are presented and compared to the theoretical calculations and Monte Carlo models.

Results are presented of the production of leading protons, neutrons and photons in deep inelastic scattering $\left(e p \rightarrow e^{\prime} p X, e p \rightarrow e^{\prime} n X, e p \rightarrow e^{\prime} \gamma X\right.$ ) as well as the leading neutron production in the photoproduction of dijets $(e p \rightarrow e j j X n)$. The forward baryon and photon results from the H1 and ZEUS Experiments are compared also with the models of the hadronic interactions of high energy Cosmic Rays. The sensitivity of the HERA data to the differences between the models is demonstrated.
\end{abstract}

\section{Introduction}

The QCD hardness scale for secondary particles production in semi-inclusive deep inelastic scattering (DIS) gradually decreases from the photon virtuality, $Q^{2}$, which determines the hard scale in the virtual photon fragmentation (current) region, to a soft hadronic scale in the proton fragmentation region. In the processes with low $Q^{2}$ (photoproduction) the hard scale of the interaction can be guaranteed by the presence in the final state of the hadronic jets or heavy quarks. Particle production at very small angles with respect to the proton beam direction (forward direction) in a process with a hard scale provides a testing ground for the theory of strong interactions in the soft regime and is important for the theoretical understanding of proton fragmentation.

Measurements of forward particles also provide important constraints for the modelling of the high energy air showers and thereby are very valuable for the understanding of high energy Cosmic Ray data [1].

In this presentation, the recent results of the $\mathrm{H} 1$ and ZEUS Collaborations of the forward proton, neutron and photon production in electron-proton and positron-proton interactions at HERA collider are reported.

\section{Leading Baryons at HERA}

In $e p$ scattering at HERA a significant fraction of events contains in the final state a proton or a neutron, which carry a large fraction $x_{L}$ of the incoming proton energy. Although a fraction of these leading baryons (LB) may result from the hadronisation of the proton remnant, the $t$-channel exchange of the colour singlet virtual particles

\footnotetext{
aArmen.Buniatyan@desy.de
}

(corresponding to the diagram shown in Figure 1) is expected to contribute significantly [2-5]. The production of the leading neutron (LN) in the virtual exchange model occurs through the exchange of isovector states, and $\pi^{+}$ exchange is expected to dominate. For the leading proton (LP) production, isoscalar exchanges also contribute, including diffractive Pomeron mediated interactions.

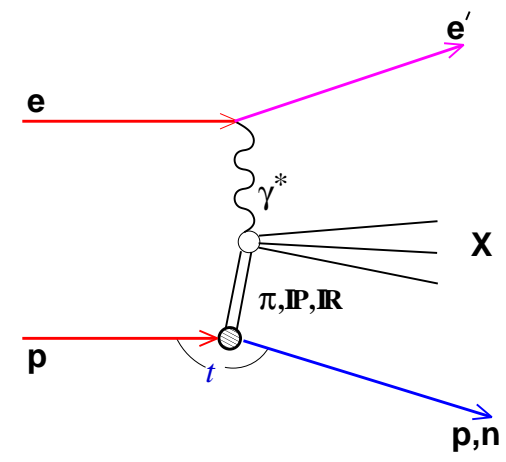

Figure 1. Leading baryon production $e p \rightarrow e X N$ via the colour singlet exchange processes.

In the simple exchange picture, the cross section is factorised into two parts (vertex factorisation): one factor describes the probability of the 'emission' of a colour neutral object from the beam proton, the other describes the scattering of virtual photon with the exchanged particle. For instance, the cross section of LN production via $\pi^{+}$-meson exchange can be written as

$$
d \sigma_{\gamma^{*} p \rightarrow n x}=f_{\pi / p}\left(x_{L}, t\right) \times d \sigma_{\gamma^{*} \pi \rightarrow X}
$$

Here $f_{\pi / p}$ is the flux of virtual pions in the proton, which is constrained from the low energy hadronic data. Such a 
reaction can thus be used to probe the partonic structure of the exchanged pion.

In order to measure the leading protons and neutrons, the H1 and ZEUS Experiments have been equipped with dedicated detectors. The protons were measured with position sensitive detectors (Roman Pots) placed along the proton beam downstream of the interaction point. The neutrons were measured with lead-scintillator forward calorimeters (FNC) at the zero-degree point; magnet apertures limited neutron detection to scattering angles less than $0.75 \mathrm{mrad}$.

\subsection{Leading Proton and Leading Neutron cross sections as a function of $x_{L}$}

The cross section of LP production in DIS normalised to the inclusive DIS cross section $\left(1 / \sigma_{t o t} \cdot d \sigma_{L P} / d x_{L}\right)$, measured as a function of the longitudinal momentum fraction $x_{L}$ of the scattered proton, is shown in Figure 2 [7]. Here $x_{L}=E_{p} / E_{p}^{\text {beam }}$, where $E_{p}^{\text {beam }}$ is the energy of a beam proton. The LP rate is approximately flat up to the diffractive peak at $x_{L} \sim 1$, where it increases by a factor of about six. In the upper part of Figure 2 the distribution is compared to the predictions of Monte Carlo (MC) models DJANGOH [8] and RAPGAP [9], which are based on standard fragmentation. These models don't reproduce the flat dependence of the cross section below the diffractive peak. The same data are compared to a Regge-based model [6] which incorporates the isovector and isoscalar exchanges and includes the Pomeron exchange for diffraction. A good de-

\section{ZEUS}

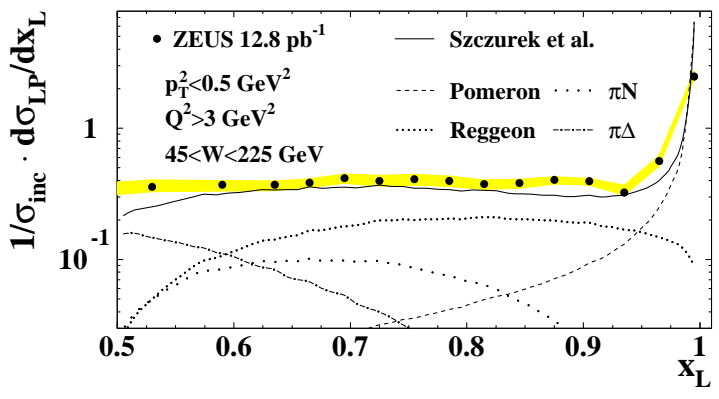

\section{ZEUS}

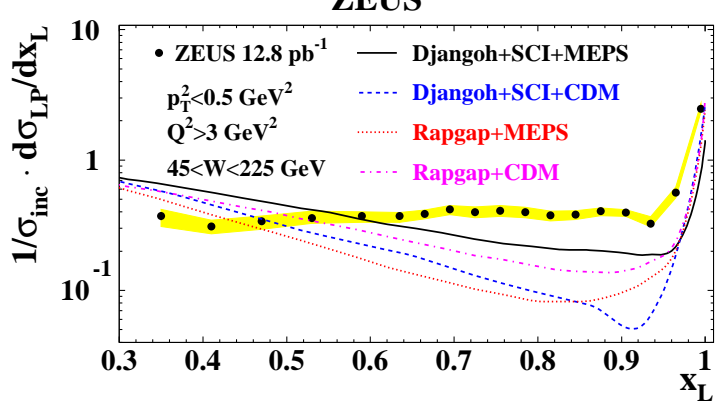

Figure 2. Normalised leading proton production cross section in DIS as a function of $x_{L}$, compared to the Monte Carlo models (up) and Regge-based model [6] (bottom).

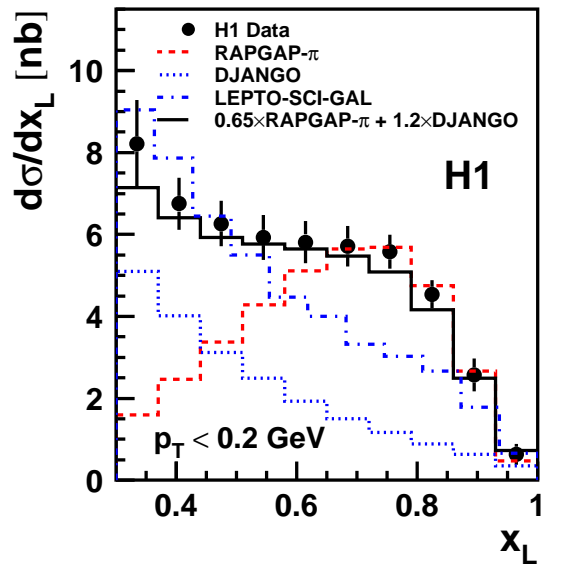

ZEUS

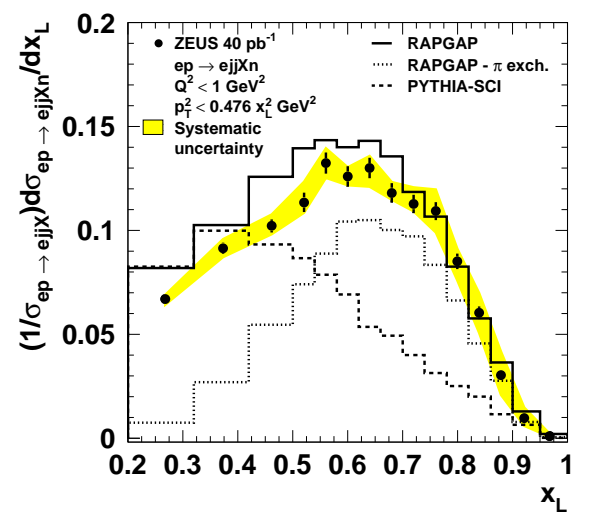

Figure 3. Differential cross section of the leading neutron production as a function of $x_{L}$ in the angular range $\theta_{n}<0.75 \mathrm{mrad}$ in DIS, in the phase space defined by the photon virtuality $6<$ $Q^{2}<100 \mathrm{GeV}^{2}$ and the inelasticity $0.02<y<0.6$ (up) and in the photoproduction of dijets in the kinematic range defined by $Q^{2}<1 \mathrm{GeV}^{2}$ and the jet transverse energies and pseudorapidities $E_{T}^{\text {jet } 1(2)}>7.5(6.5) \mathrm{GeV},-1.5<\eta^{\text {jet } 1,2}<2.5$ (bottom). Predictions of the MC simulations are compared with the measurements.

scription of the $x_{L}$ distribution is obtained by adding a substantial contribution of isoscalar Reggeon exchanges, which turn out to be the dominant processes below the diffractive peak.

Figure 3 shows the cross sections of LN production as a function of $x_{L}$ in DIS [10] and in the photoproduction of dijets [11]. The DIS measurement is compared with the prediction of RAPGAP MC [9], which here generates exclusively the $\pi^{+}$-exchange process, and the standard fragmentation model is simulated with DJANGOH [8]. For the comparison with the photoproduction data, the RAPGAP simulation incorporates the standard fragmentation and the $\pi^{+}$-exchange processes. The photoproduction measurement is also compared with the PYTHIA MC [12], which includes the simulation of soft colour interactions (SCI) [13], in which the production of diffraction-like configurations is enhanced via non-perturbative colour rearrangements between the outgoing partons. Both distribu- 
tions are well described by the combination of the standard fragmentation and $\pi^{+}$-exchange models. At large $x_{L}$ values the contribution from $\pi^{+}$-exchange dominates.

Figure 4 compares the $x_{L}$ distributions for the leading protons and neutrons restricted to the same $p_{T}$ range $\left(p_{T}^{2}<0.04 \mathrm{GeV}^{2}\right)$. If the LP production proceeded only through isovector exchange, as LN production must, there would be half as many LP as LN. The data instead has approximately twice as many LP as LN. Thus, exchanges of particles with different isospins such as isoscalars must be invoked for LP production.

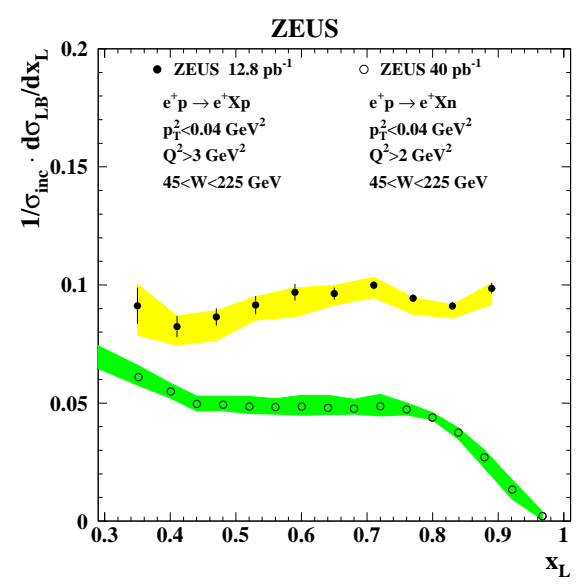

Figure 4. The rate $1 / \sigma_{i n c} d \sigma_{L B} / d x_{L}$ for the leading proton (dots) and the leading neutron (open circles) production in DIS restricted to $p_{T}^{2}<0.04 \mathrm{GeV}^{2}$ range.

\subsection{Leading Neutron $x_{L}$ and $p_{T}^{2}$ cross sections in DIS}

The measurement of the double differential cross section of LN production in DIS as a function of $x_{L}$ and the squared transverse momentum $p_{T}^{2}$ of the neutron is shown in Figure 5 [14]. The best description of the data is achieved by the combination of the standard fragmentation and $\pi^{+}$-exchange models, similar to the single differential $x_{L}$ distribution shown in Figure 3. Assuming the dominance of the $\pi^{+}$-exchange mechanism for LN production the shape of $p_{T}^{2}$ (or the squared four momentum transfer at proton vertex $t$ ) distribution is directly related to the pion flux $f_{\pi / p}$ (see Eq.1). Since the $p_{T}^{2}$ distributions from Figure 5 have almost exponential shapes in all $x_{L}$ bins, they can be fitted by an exponential function $a\left(x_{L}\right) \exp \left(-b\left(x_{L}\right) p_{T}^{2}\right)$. The obtained values of the $p_{T}^{2}$ slope parameters in $x_{L}$ bins, $b\left(x_{L}\right)$, are shown in Figure 6 together with the slopes obtained using the different parameterisations of the pion flux $[3-5,15,16]$. Most of the shown predictions describe the data within uncertainties.

\subsection{Leading Baryon production rate in DIS}

It is instructive to investigate the relative yield of the leading baryon production as a function of the DIS kinematic parameters, with respect to the inclusive DIS events.

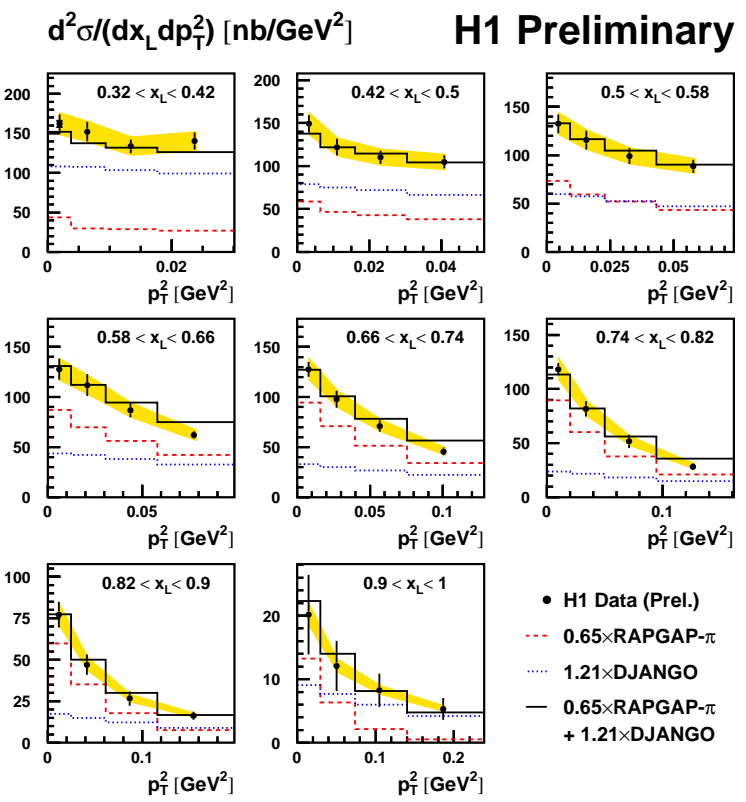

Figure 5. Double differential cross section of the leading neutron production in DIS as a function of $p_{T}^{2}$ and $x_{L}$ of the neutron. Data are compared with predictions of the RAPGAP pion exchange and DJANGOH MC simulations as well as combination of those two simulations.

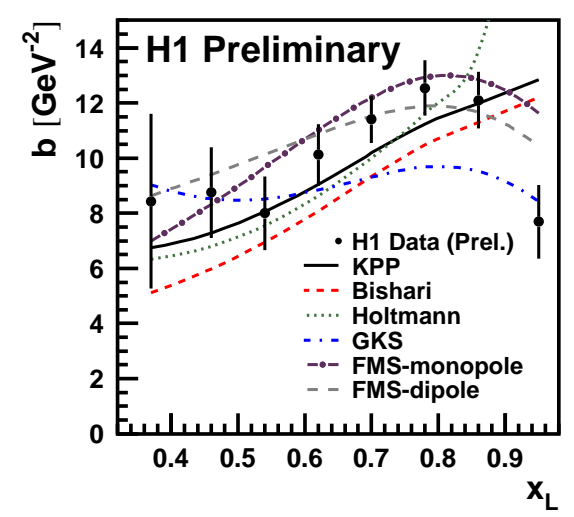

Figure 6. $b$-slopes of the $p_{T}^{2}$ distributions of the leading neutrons in DIS compared with the different parameterisations of pion flux.

The hypothesis of limiting fragmentation suggests that the production of leading baryons in the proton fragmentation region is independent of the photon virtuality $Q^{2}$ and Bjorken $x$ variable. Figure 7 displays the LP production rate $r^{L P}$ as a function of $x$ in bins $Q^{2}$ and Figure 8 shows the ratio of semi-inclusive structure function of the leading neutron production $F_{2}^{L N}$ to the proton structure function $F_{2}$ (i.e. the LN production rate) as a function of $Q^{2}$ in bins of 
$x$ and $x_{L}$. The ratios are almost independent of $x$ and $Q^{2}$, as expected from the hypothesis of limiting fragmentation.

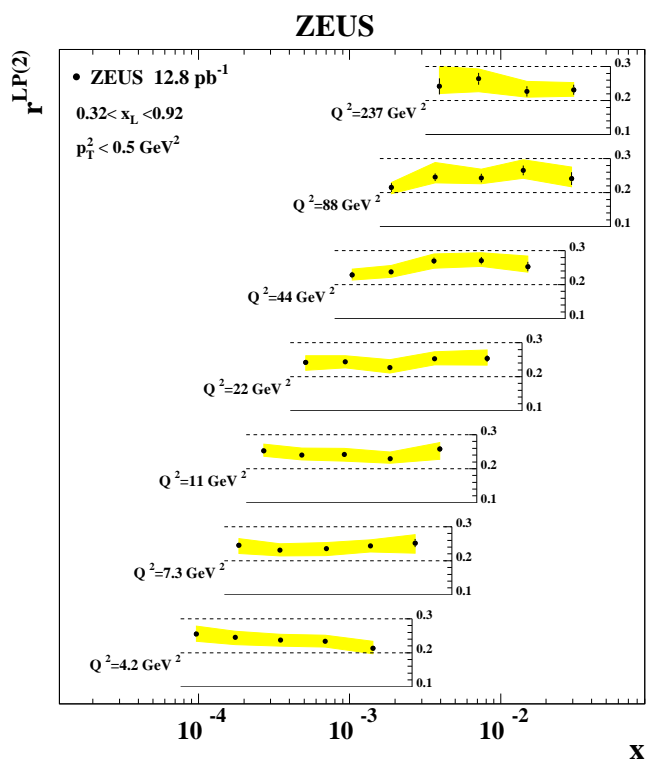

Figure 7. The leading proton production rate in DIS $r^{L P}$ as a function of Bjorken $x$ in bins $Q^{2}$.

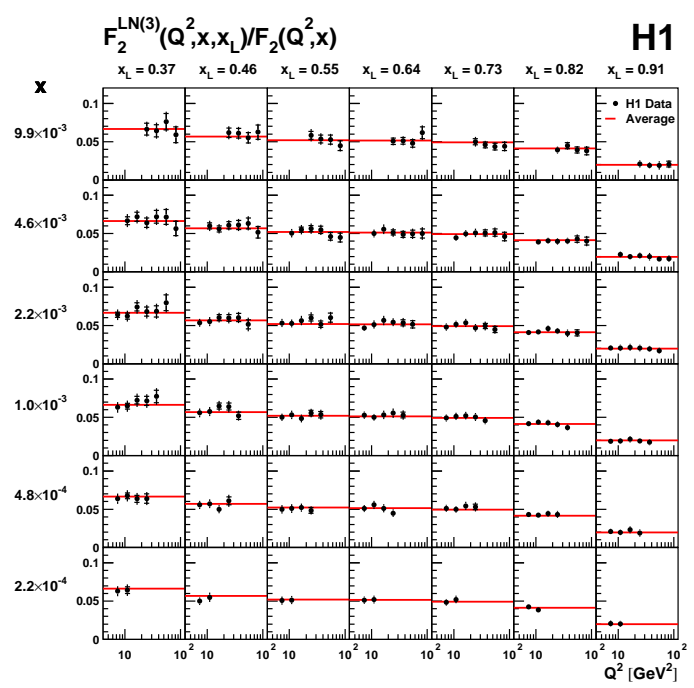

Figure 8. The ratio of the semi-inclusive structure function $F_{2}^{L N}\left(Q^{2}, x, x_{L}\right)$ to the proton structure function $F_{2}\left(Q^{2}, x\right)$.

\subsection{Photoproduction of dijets with LN}

The photoproduction of dijets in events with LN is measured in the kinematic range defined by $Q^{2}<1 \mathrm{GeV}^{2}$ and the jet transverse energies and pseudorapidities $E_{T}^{\text {jet } 1(2)}>$ 7.5 (6.5) $\mathrm{GeV},-1.5<\eta^{\text {jet } 1,2}<2.5$. The differential cross sections as a function of the event variables $x_{\gamma}^{O B S}, x_{p}^{O B S}$ and $W$ are presented in Figure 9. Here, $x_{\gamma}^{O B S}$ and $x_{p}^{O B S}$ are respectively the fractions of the four-momenta of the photon and the proton, which participate in the hard interaction, and $W$ is the centre-of-mass energy of the $\gamma p$ system. Also shown are the cross sections of inclusive photoproduction of dijets and the ratios of the dijet cross sections calculated with the requirement of $\mathrm{LN}$ in the final state to the inclusive dijet cross sections. As one can see from the ratio distributions, the LN sample has a significantly smaller contribution at low $x_{\gamma}^{O B S}$. The cross sections are roughly flat as a function of $W$; the yield exhibits a decrease with increasing $W$ and $x_{p}^{O B S}$. For the LN sample, RAPGAP overestimates the cross section at low $x_{\gamma}^{O B S}$ while PYTHIASCI underestimates the cross section at high $x_{\gamma}^{O B S}$. Neither model can reproduce the dependence of the neutron yield on $x_{\gamma}^{O B S}$ and $W$. The RAPGAP model predicts a small decrease of the neutron yield with $x_{p}^{O B S}$, which however is less pronounced than in the data. This dependence of the neutron yield indicates a violation of vertex factorisation.

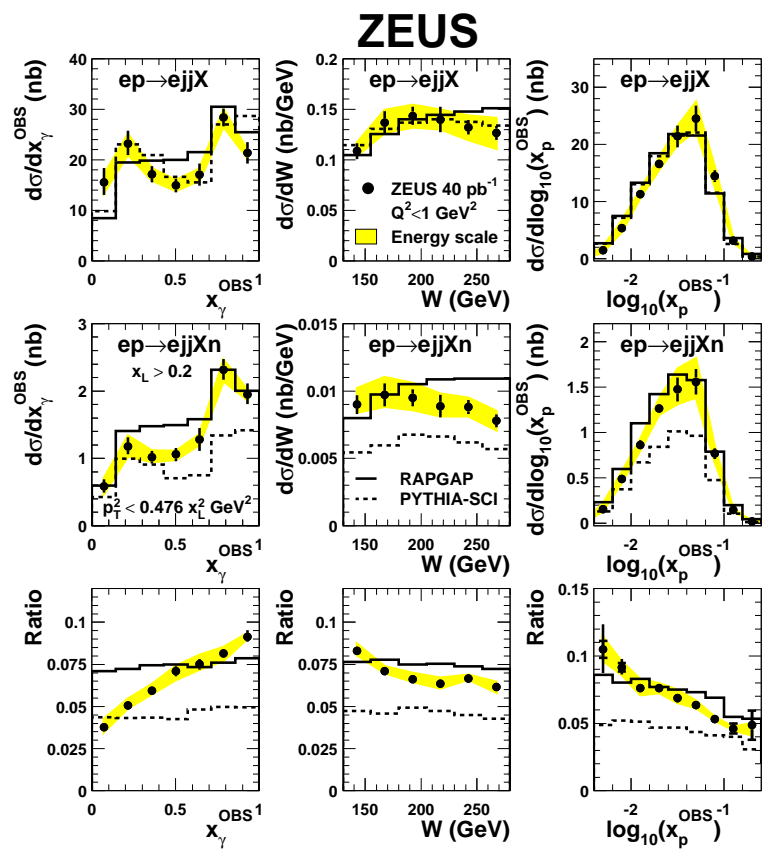

Figure 9. Differential cross sections of dijet photoproduction with leading neutrons and inclusive dijet photoproduction as a function of $x_{\gamma}^{O B S}, W$ and $\log _{10}\left(x_{p}^{O B S}\right)$. The ratios between cross sections are also given.

\subsection{Comparison of Forward Baryon results from HERA with Cosmic Ray interaction models}

The measurements of forward particles at HERA may provide valuable information for the physics of ultra-high energy Cosmic Rays (CR). The models which are used for $\mathrm{CR}$ analyses can be used to make predictions for HERA kinematics, which then can be compared to the experimental measurements. Here the comparisons are presented of HERA measurements with the several models of hadronic interaction developed for the simulation of air shower cascades: EPOS [17], QGSJET 01 [18, 19], QGSJET II [20, 21] and SIBYLL [22, 23]. 
Comparison of the leading proton and the leading neutron spectra measured at HERA with the predictions of the CR models [24] are shown in Figure 10. While all tested models give reasonable description of the leading proton data, they differ significantly in the predictions for the leading neutrons. Thus, HERA forward particle measurements are sensitive to the differences between the models and can be used for further tuning of model parameters.
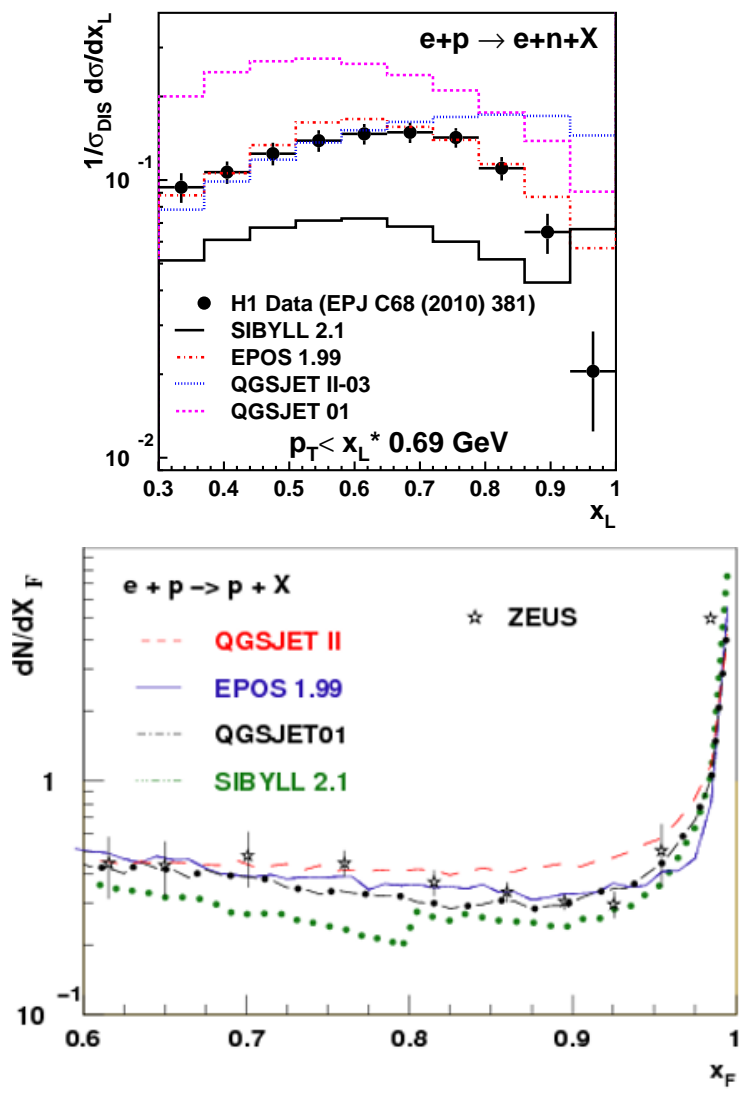

Figure 10. Comparison of the leading neutron (up) and the leading proton (bottom) $x_{L}$ distributions measured in DIS at HERA with the predictions of the Cosmic Ray interaction models. (The LN data from [10] are normalised to the total inclusive DIS cross section in the quoted kinematic range.)

\section{Forward photon spectra in DIS}

The production of photons at very small angles with respect to the proton beam direction is studied in DIS at HERA [25] by the H1 Experiment. The forward photons are measured in the electromagnetic section of the FNC calorimeter.

For the most energetic forward photon in the pseudorapidity range $\eta>7.9$ (leading photon) the cross sections are presented as a function of its transverse momentum $p_{T}^{\text {lead }}$ and longitudinal momentum fraction of the incoming proton $x_{L}^{\text {lead }}$. In Figure 11 the data are compared with DJANGOH [8] MC model predictions, in which higher order QCD effects are simulated using leading log parton showers as implemented in LEPTO [26], or using the Colour
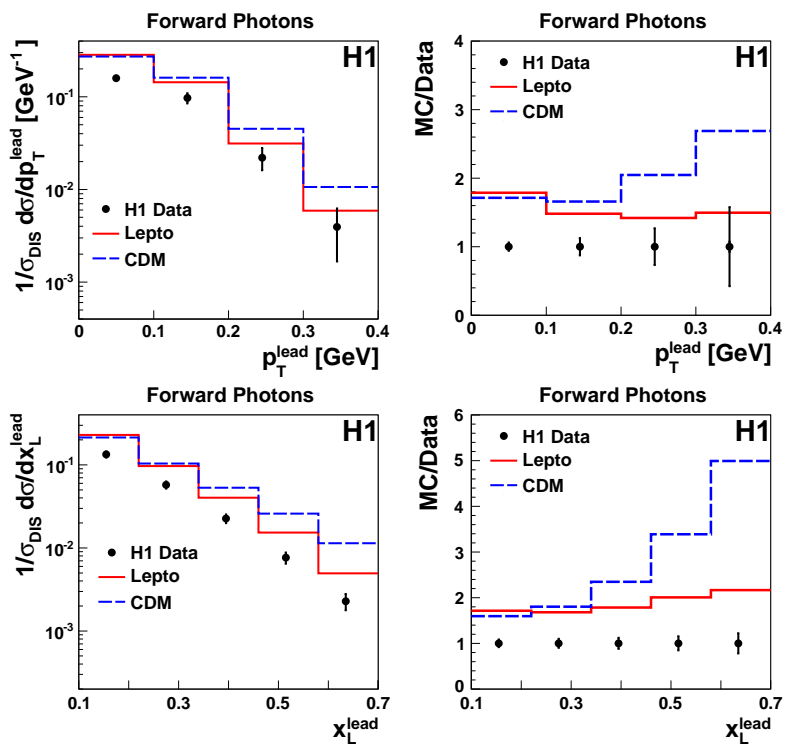

Figure 11. The normalised cross sections for the production of forward photons in the pseudorapidity range $\eta>7.9$ in DIS in the kinematic region $6<Q^{2}<100 \mathrm{GeV}^{2}$ and $0.05<y<0.6$ as a function of the transverse momentum $p_{T}^{\text {lead }}$ and longitudinal momentum fractions $x_{L}^{\text {lead }}$ of leading photon. The ratios of the measured cross sections to the LEPTO and CDM MC model predictions are shown in the right sides of the plots.

Dipole Model (CDM) as implemented in ARIADNE [27]. In Figure 12 the measurements are also compared with the predictions of Cosmic Ray hadronic interaction models. All models overestimate the total rate of forward photons. The shapes of measured distributions are well described by LEPTO. CDM predicts harder $x_{L}$ and $p_{T}$ spectra. The QGSJET models predict slightly softer spectra, while the EPOS and SIBYLL models predict harder $x_{L}$ spectra, but reasonably describe the shape of $p_{T}$ distribution.

The normalised forward photon production cross section as a function of virtual photon-proton centre-of-mass energy, $W$, is shown in Figure 13. It is determined as the ratio of the cross section of forward photon production to the inclusive DIS cross section in each $W$ bin. Within uncertainties, the measured ratio is consistent with a constant value, suggesting that the shapes of the $W$ distributions are similar for inclusive DIS events and events which contain forward photons. The measurements are compared with the MC predictions of DIS models and the models of hadronic interactions (EPOS, SIBYLL and two versions of QGSJET). The models indicate slight $W$ dependence of the ratios.

\subsection{Analysis of Feynman scaling with the forward photons}

The forward photon production has been studied also as a function of Feynman- $x$ variable. This variable is defined as $x_{F}=2 p_{\|}^{*} / W$, where $p_{\|}^{*}$ is the longitudinal momentum of forward photon in the CMS frame of the virtual photon - proton system. This measurement allows the Feyn- 

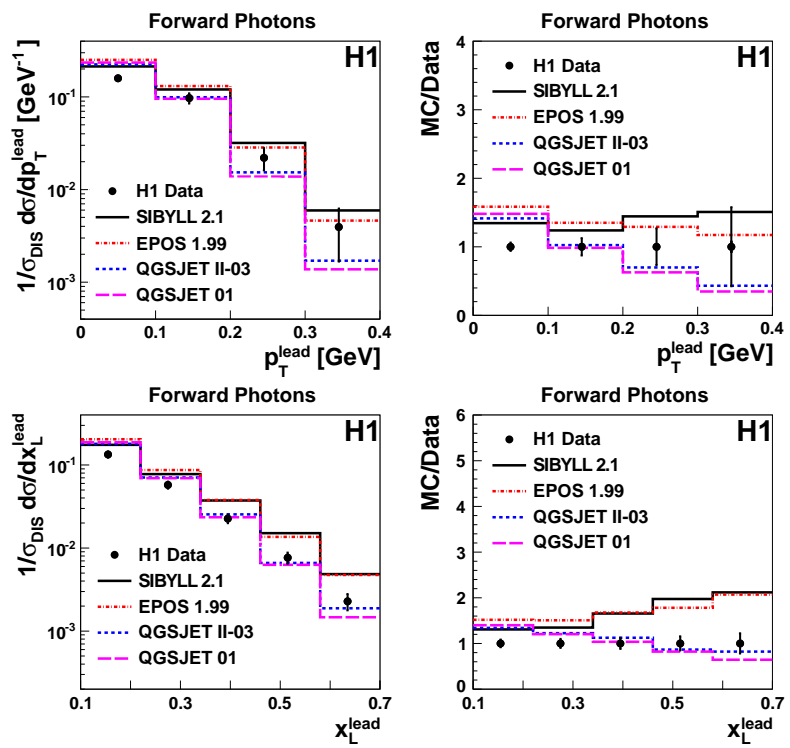

Figure 12. The normalised cross sections for the production of forward photons in the pseudorapidity range $\eta>7.9$ in DIS in the kinematic region $6<Q^{2}<100 \mathrm{GeV}^{2}$ and $0.05<y<0.6$ as a function of the transverse momentum $p_{T}^{\text {lead }}$ and longitudinal momentum fractions $x_{L}^{\text {lead }}$ of leading photon. The ratios of the measured cross sections to Cosmic Ray hadronic interaction model predictions are shown in the right sides of the plots.

man scaling, which predicts the independence of $x_{F}$ distributions from the total centre-of-mass energy $W$, to be tested. Figure 14 shows the measured $x_{F}$ cross sections for forward photons in three $W$ intervals, normalised to the inclusive DIS cross section in corresponding $W$ intervals. The measurements are compared to the predictions of Cosmic Ray interaction models. The differences between the measurements and predictions have similar behaviour as for $x_{L}^{\text {lead }}$ distributions shown above in Figure 12. In order to study the energy dependence of $x_{F}$ distributions in details, the ratios are made of the $x_{F}$ cross sections in different $W$ intervals. The ratios of the $x_{F}$ cross sections in the ranges $130<W<190 \mathrm{GeV}$ and $190<W<250 \mathrm{GeV}$ to that in the range $70<W<130 \mathrm{GeV}$, are shown in the upper and lower plots of Figure 15, respectively. The ratio distributions for the data are consistent with the unity within uncertainties, confirming the validity of Feynman scaling for forward photon production in the measured kinematic range. A similar behaviour is predicted by CDM and LEPTO MC models. The CR models indicate the deviations from the scaling, i.e. the rate of forward photons becomes lower with increasing energy $W$.

\section{Summary}

The production of the forward protons, neutrons and photons has been studied in DIS, and the production of leading neutrons in the photoproduction of dijets.

The contributions from the fragmentation processes and from the exchange of colour-neutral particles are required to describe the leading baryon data. The $p_{T}^{2}$ spec-
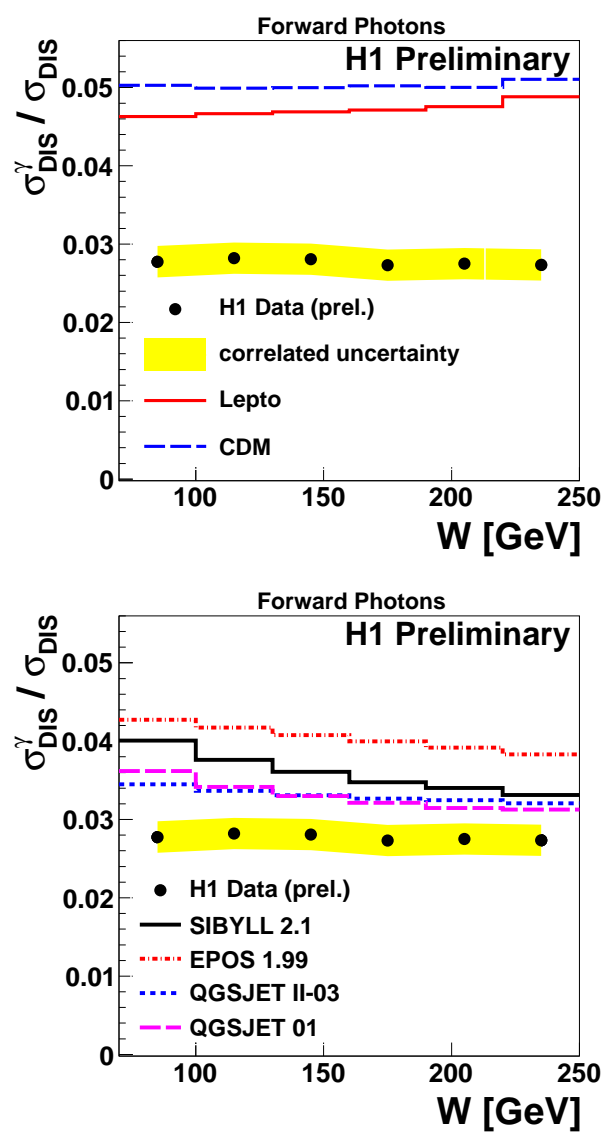

Figure 13. The fraction of DIS events with forward photons in the kinematic region $6<Q^{2}<100 \mathrm{GeV}^{2}$ and $0.05<y<0.6$ and the pseudorapidity of the photon $\eta>7.9$. as a function of $W$. The predictions of MC models are compared to the measurements.

tra of the forward neutrons show sensitivity to the pion flux parameterisations. The hadronic interaction models of Cosmic Rays describe well the leading proton data, but differ in the predictions for leading neutron energy spectra.

The production of the forward photons has been studied in DIS as a function of the longitudinal momentum fraction $x_{L}$, the transverse momentum $p_{T}$ and Feynman$x$ variable. Predictions of all tested Monte Carlo models overestimate the rate of forward photon production. The models predict different spectra in $x_{L}$ and $p_{T}$; none of them can describe the forward photon data in rate and in shape. Within the measured kinematic range the $x_{F}$ spectra are insensitive to the virtual photon-proton centre-of-mass energy $W$, confirming the validity of Feynman scaling.

The present measurements may lead to further understanding of proton fragmentation in collider and Cosmic Ray experiments.

\section{Acknowledgements}

Finally, I wish to thank the organisers for the excellent organisation and atmosphere of the conference which I enjoyed very much. 

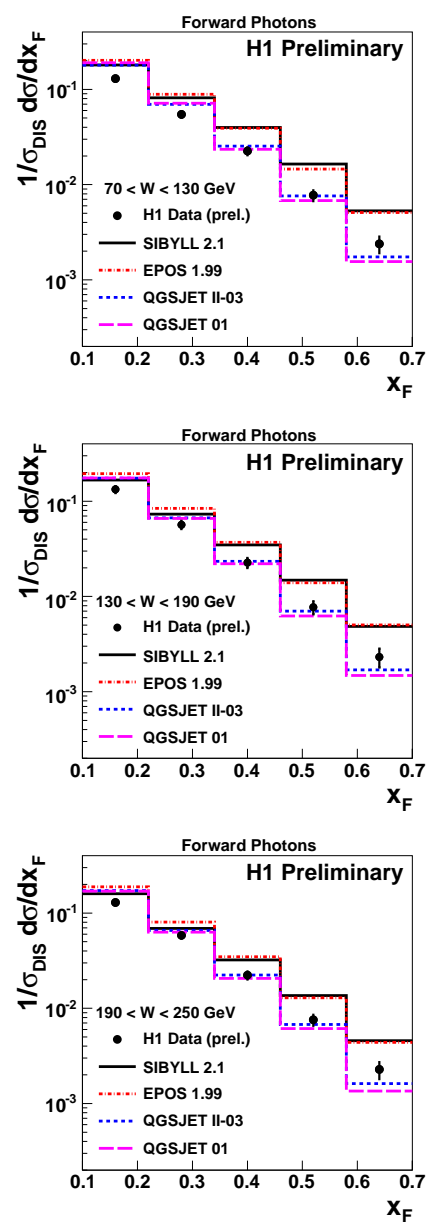

Figure 14. Normalised cross sections of forward photon production in DIS as a function of $x_{F}$ in the region $\eta>7.9$, $6<Q^{2}<100 \mathrm{GeV}^{2}$ a and $0.05<y<0.6$ in three $W$ intervals. The data are compared to predictions of Cosmic Ray hadronic interaction models.

\section{References}

[1] A. Bunyatyan, A. Cooper-Sarkar, C. Diaconu, R. Engel, C. Kiesling et al., pp.567-581 (2009)

[2] J.D. Sullivan, Phys. Rev. D5, 1732 (1972)

[3] M. Bishari, Phys. Lett. B38, 510 (1972)

[4] H. Holtmann et al., Phys. Lett. B338, 363 (1994)

[5] B. Kopeliovich, B. Povh, I. Potashnikova, Z. Phys. C73, 125 (1996), hep-ph/9601291

[6] A. Szczurek, N.N. Nikolaev, J. Speth, Phys. Lett. B428, 383 (1998), hep-ph/9712261

[7] S. Chekanov et al. (ZEUS Collaboration), JHEP 06, 074 (2009), 0812 . 2416

[8] K. Charchula, G.A. Schuler, H. Spiesberger, Comput. Phys. Commun. 81, 381 (1994), DJANGOH 1.4

[9] H. Jung, Comp. Phys. Commun. 86, 147 (1995), RAPGAP 3.1

[10] F.D. Aaron et al. (H1 Collaboration), Eur. Phys. J. C68, 381 (2010), 1001.0532

[11] S. Chekanov et al. (ZEUS Collaboration), Nucl. Phys. B827, 1 (2010), 0909. 3032
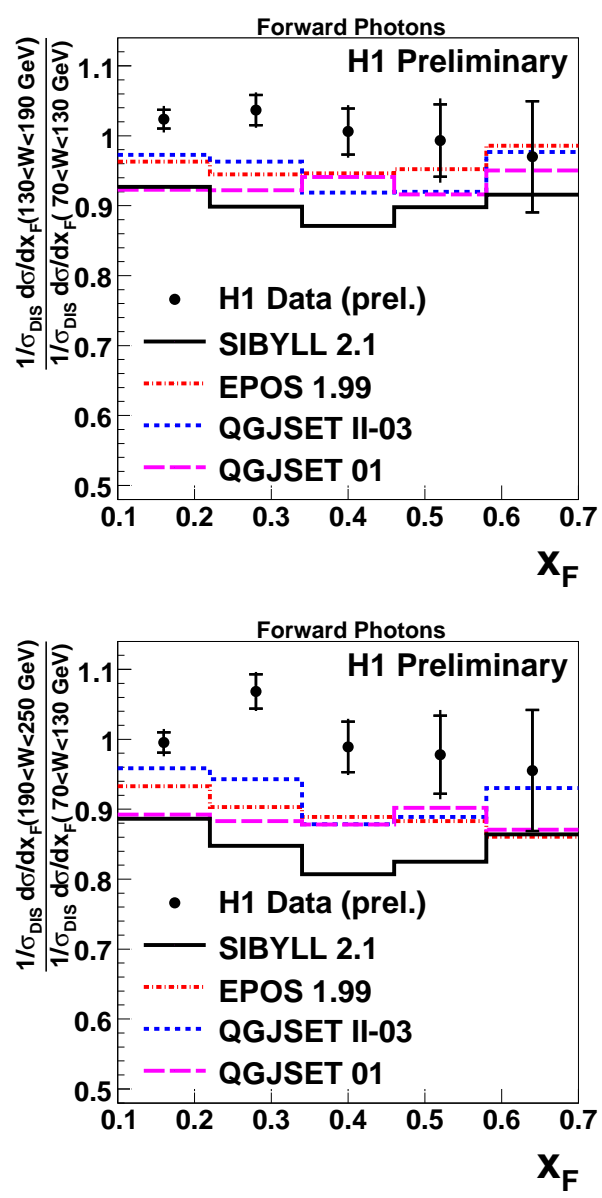

Figure 15. Ratios of normalised cross sections of forward photons production in DIS from the different $W$ intervals shown in Figure 14 as a function of $x_{F}$. Upper plot shows the ratio of the cross section in $130<W<190 \mathrm{GeV}$ interval to the cross section in $70<W<130 \mathrm{GeV}$ interval. The lower figure shows the ratio of the cross section in $190<W<250 \mathrm{GeV}$ interval to the cross section in $70<W<130 \mathrm{GeV}$ interval. Predictions of Cosmic Ray hadronic interaction models are compared to the measurement.

[12] M.S. Sjostrand, T., P.Z. Skands, JHEP 05, 026 (2006), hep-ph/0603175

[13] A. Edin, G. Ingelman, J. Rathsman, Phys. Lett. B366, 371 (1996), hep-ph/9508386

[14] H1 Collaboration, Leading Neutron $p_{T}$ distribution, H1prelim-10-113 (2010), https://wwwh1.desy.de/h1/www/publications/htmlsplit/H1 prelim10-113.long.html

[15] K.J. Golec-Biernat, J. Kwiecinski, A. Szczurek, Phys. Rev. D56, 3955 (1997), hep-ph/9701254

[16] L.L. Frankfurt, L. Mankiewicz, M.I. Strikman, Z. Phys. A334, 343 (1989)

[17] K. Werner, F.M. Liu, T. Pierog, Phys. Rev. C74, 044902 (2006), hep-ph/0506232

[18] N.N. Kalmykov, S.S. Ostapchenko, Phys. Atom. Nucl. 56, 346 (1993)

[19] N.N. Kalmykov, S.S. Ostapchenko, A.I. Pavlov, Nucl. Phys. Proc. Suppl. 52B, 17 (1997) 
[20] S.S. Ostapchenko, Phys. Rev. D74, 014026 (2006), hep-ph/0505259

[21] S.S. Ostapchenko, AIP Conf. Proc. 928, 118 (2007), 0706. 3784

[22] J. Engel, T.K. Gaisser, T. Stanev, P. Lipari, Phys. Rev. D46, 5013 (1992)

[23] E.J. Ahn, R. Engel, T.K. Gaisser, P. Lipari, T. Stanev, Phys. Rev. D80, 094003 (2009), 0906. 4113
[24] Monte Carlo simulation of Cosmic Ray hadronic interaction models provided by Tanguy Pierog

[25] F. Aaron et al. (H1 Collaboration), Eur.Phys.J. C71, 1771 (2011), 1106.5944

[26] G. Ingelman, A. Edin, J. Rathsman, Comput. Phys. Commun. 101, 108 (1997), hep-ph/9605286

[27] L. Lönnblad, Comput. Phys. Commun. 71, 15 (1992), ARIADNE 4.10 